\title{
Stochastic Process of Pit Generation of Aluminum
}

\author{
Toshio Shibata* and Masatoshi Sudo
}

Received August 28, 1989 ; Accepted November 24, 1989

\begin{abstract}
Pit generation of aluminum in $3.5 \%$ aqueous $\mathrm{NaC1}$ solution has been analyzed based on a stochastic theory. The distributions of pitting potential measured by a potential sweep method and induction time for pit generation at a constant potential were found to be rationally explained by assuming the stochastic model of a plural combination of an elemental Poisson type stochastic process.
\end{abstract}

\section{INTRODUCTION}

Aluminum and its alloys show a remarkable corrosion resistance in air or in water environments due to formation of stable thin oxide films on the surface. It is well known, however, that halogen ions in environments causes a breakdown of the film, leading to generation of pits which eventually penetrate into the substrate. Many studies have been reported for developing corrosion resistant aluminum alloys as well as for clarifying the mechanism of pit formation, but most of them have been made on the basis of a deterministic point of view.

Our previous studies on pit generatign processes for stainless steels 1 , 2) demonstrated that a statistical and probabilistic point of view is highly important for analyzing the process because experimental data of pitting potential as well as induction time for

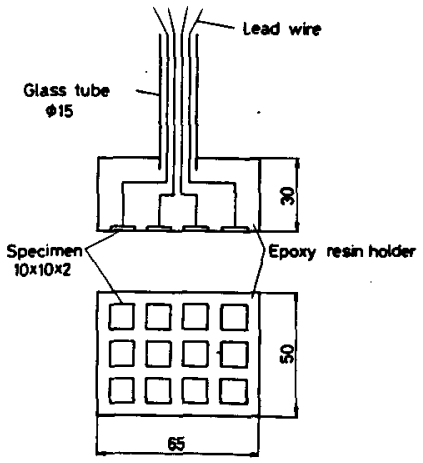

Fig.1 Multiple specimens holder

* Depertment of Materials Science and Processing, Faculty of Engineering, Osaka University,

Yamadaoka 2-1, Suita 0saka 565 JAPAN

Key Words: Aluminum, NaCl solution, Pit Generation, Stochastic process. pit generation always exhibits a substantial variation.

This study aims to analyze a pit generation process of aluminum in a sodium chloride solution as a stochastic process for establishing an exact stochastic model.

\section{EXPERTMENTAL}

Small square plates of specimen $(10 \times 10 \times 2$ $\mathrm{mm}$ ) were obtained from a sheet of commercial pure aluminum (99.99 mass\%), impurity levels of which were as follows, $\mathrm{Si}: 6, \mathrm{Fe}: 6, \mathrm{Cu}: 46, \mathrm{Mg}:<1$ in $10^{-4}$ mass\%. For the pit generation experiment, 12 specimens were molded into an epoxy resin holder as shown in Fig.1. Each specimen was screwed by a bolt of $0.8 \mathrm{~mm}$ diameter, which was soldered with a lead wire. Surface of the specimens was polished with $0 / 6$ emery paper and subsequently with wet alumina powder immediately before the experiment. A multichannel pitting corrosion testing apparatus, the principle of which was shown in Fig.2, was used for measuring pitting potential by a potential sweep method and induction time for pit

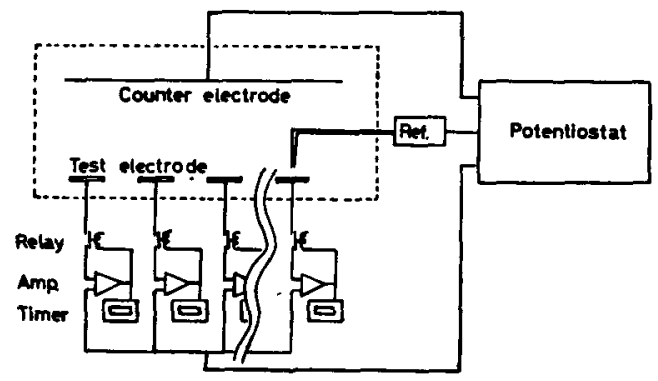

Fig.2 Principle of the multichannel pitting corrosion testing apparatus which can determine 12 values of pitting potential or induction time in an identical condition. 
generation at a constant potential. With this apparatus and 12 specimens molded in the holder shown in Fig.1, 12 values of pitting potential or induction time were obtained at an identical condition and repetition of the same experiment yielded a number of values, from which the probability distribution was determined. A11 pit generation experiments were done in 3.5 mass \% NaCl solution which was deaerated by purified nitrogen gas and at temperatures from $288 \mathrm{~K}$ to $323 \mathrm{~K}$. Potential was measured referring to $\mathrm{Ag} / \mathrm{AgCl}$ electrode and converted into the standard hydrogen electrode scale(V vs. SHE).

\section{Anodic polarization curve}

Anodic polarization curves of aluminum measured in 3.5 mass\% $\mathrm{NaCl}$ solution at various temperatures were shown in Fig.3. Curves were measured by the ${ }_{4}$ potential sweep method with $3.47 \times 10^{-4} \mathrm{~V} \mathrm{~s} \mathrm{~s}^{-1}$ starting from corrosion potential to noble potential. In the less noble potential region, anodic current increased slowly with potential, but suddenly rose at around $-0.5 \mathrm{~V}$ due to pit generation.

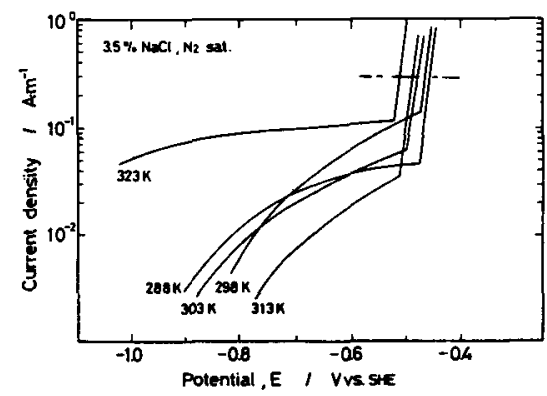

Fig.3 Polarization curves of aluminum in 3.5 mass\% $\mathrm{NaCl}$ solution at various temperatures.

This pit generation potential is usually called pitting potential. By the definition, pitting potential is the lowest potential just to detect an initial current increase due to nucleation of a pit. Pitting potential thus defined, however, depends on a sensitivity for measuring the current increase, so that a criterion using a constant current density is conventionally used to decide pitting potential. In this study, pitting potential was defined conventionally as a potential at which $0.4 \mathrm{~A} \mathrm{~m}^{-2}$ of anodic current was observed as shown in Fig.3. If this critical value of current is used, the difference between the exact pitting potential and the conventional one is quite small because of a steep increase of anodic current was occurred beyond the pit generation potential. From a preliminary experiment, pitting potential was found to be sensitive for the pretreatment potential in the less noble potential region, so that, immediately after immersion, the pretreatment was given for $600 \mathrm{~s}$ at the constant potential which was just $0.04 \mathrm{~V}$ below a critical potential mentioned later.

\section{Distribution of pitting potential}

The distributions of pitting potential measured with the constant potential sweep velocity indicated in the figure were plotted in normal probability paper in Fig.4. Cumulative probability, Q, shown in a vertical abscissa was calculated by a mean rank method, that is, $Q=i /(1+N)$, where $i$ is the order in the total number of $\mathrm{N}$ of measured pitting potential.

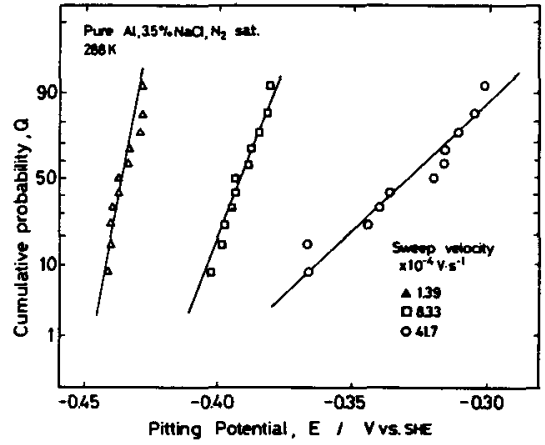

Fig.4 Distributions of pitting potential plotted in normal probability paper.

Linear lines suggests that the distribution of pitting potential obeys the normal probability distribution and the median of the distribution, $E_{\text {, }}$, was decided at $Q=50 \%$. As can be Piten in Fig.4, the slope of lines which is proportional to the inverse of standard deviation was highly dependent on the sweep velocity.

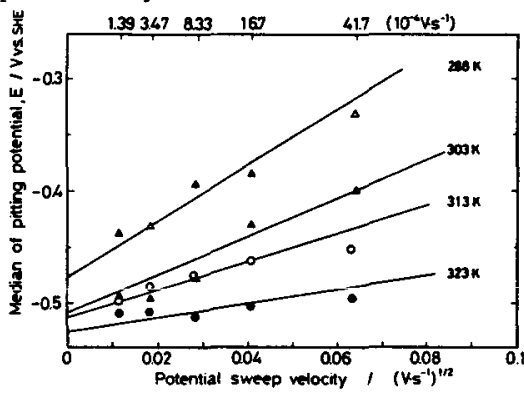

Fig. 5 Median of the distribution of pitting potential as a function of the square root of potential sweep velocity. 
Also the median of pitting potential was found to depend on the potential sweep velocity. As can be seen in Fig.5, the relation between $E_{\text {itio }}$ and the sweep velocity, $v$, was expressed as

$E_{p} \frac{i t}{\alpha}=(v / \alpha)$
where ${ }^{\prime}$ is a constant which can be also determined by the analysis of the distribution of induction time described later, and $\mathrm{E}$ is a critical pitting potential which is the lowest pitting potential realized at this condition.

\section{Distribution of induction time}

When the potential of the specimen is shifted with a step function from the pretreatment potential in the less noble potential region to a constant potential beyond $E$, pits nucleate and anodic current increases after an induction time. Induction time was found to be also a statistical variables, an example of the histogram being illustrated in Fig.6. The shape of the histogram was quite similar to what had been observed for pit generatign of stainless steel 5 and of aluminum reported by Nguen and Foley.

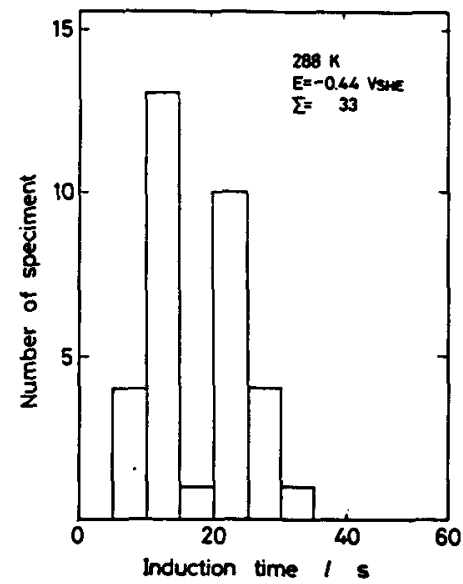

Fig.6 An example of the histgram of induction time.

\section{DISCUSSION}

As discussed in the previous paper 4$), 5$ ), a distorted distribution of induction time shown in Fig. 6 corresponds basically to an exponential probability distribution which is deduced from a Poisson, or birth stochastic process. The elemental birth stochastic process of pit generation is described by the following equation:

$$
\mathrm{dP}(\mathrm{t}) / \mathrm{dt}=-\lambda \mathrm{P}(\mathrm{t})
$$

where $\lambda$ is a pit generation rate or a transition probability from a no-pit state to a pit generated state and $P(t)$ is a survival probability which is given by $P(t)=1-i /(1+N)$, where $i$ is the order of the specimen which changes from the no-pit state to the pit generated state after time, $t$, and $N$ is the total number of the specimen examined. In the experiment, there observed no probability of pit generation within first few seconds, and then the above time not to nucleate a pit is defined as incubation time, $t_{0}$. This means that $P(t)$ for $t \leqq t$ is equal to unity. By considering the above condition of $P(t)=1$ for $t \leqq t$, integration of eq.(2) gives the survival probability for the elemental stochastic process as a function of time:

$$
P(t)=\exp \left(-\lambda\left(t-t_{0}\right)\right)
$$

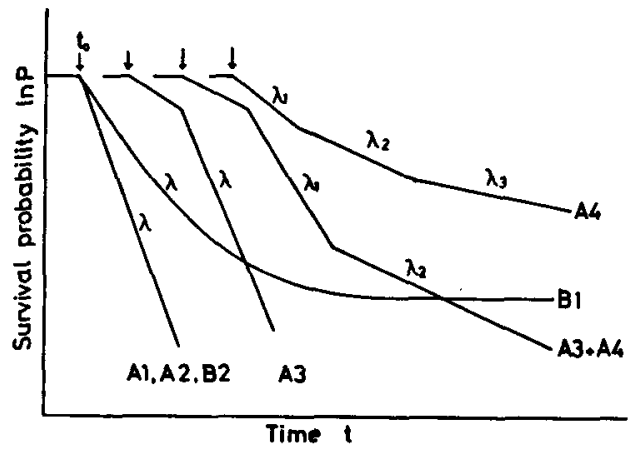

Fig.7 Schematic illustration of 1 n $\mathrm{P}$ vs. time curves expected for various stochastic models.

Table 1 Analytical expressions of the survival probability function for various stochastic models.

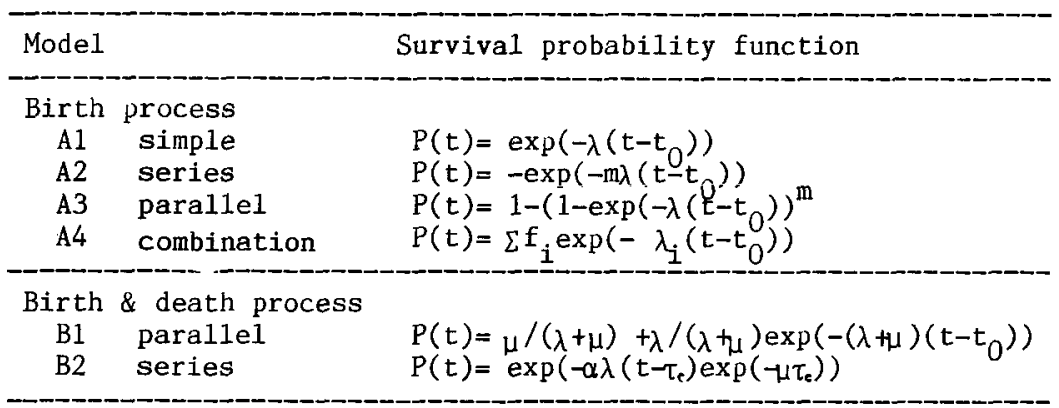


Parallel and series combinations of the above elemental stochastic process had producge a more complex stochastic

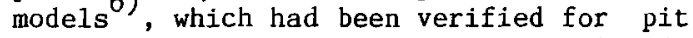
generatign of stainless steels in chloride solution ${ }^{2}$. In addition, the birth and death stochastic model had been found to fit more satisfactory for the pit generation and pịt repassivation process of stainless steels ${ }^{2}$. By solving the differential equation for the above mentioned models, various types of the survival probability function, $P(t)$, were formulated ${ }^{b}$. The analytical expressions for the survival probability function thus formulated are sumnarized in Table 1 and schematic illustration of the corresponding $\ln (\mathrm{P}(\mathrm{r}))$ vs. $t$ curves for 6yarjous models are shown in Fig. $7^{6) \text { If }}$. It should be noted that the curve for a definite model shows a characteristic shape, so that an exact model could be decided by testins whether the experimental data fits or not to the curve deduced from an assumed model.

In the present study, several models shown in Table 1 were examined and finally a parallel model was found to be well fitted to the experimental data. This model assumes that pitting events proceed with the parallel combination of $m$ elemental stochastic processes and then the probability for the pit generation process, which is given by $1-P(t)$, has to be equal to the product of the probability of pit generation of the constituent elemental process which is given by 1$P_{i}(t)$. That is,

$$
1-P(t)=\Pi\left(1-P_{i}(t)\right)
$$

When each process is assumed to be identical and have the same $\lambda$ and $t_{0}$, $P_{i}(t)$ in eq. (4) is replaced by eq. (3) and eq. (4) simply reduces to the following equation of (5)

$$
P(t)=1-\left(1-\exp \left(-\lambda\left(t-t_{0}\right)\right)^{m}\right.
$$

Schematic illustration of the $\ln (P(t))$ vs. $t$ curve given by eq. (5) is shown as $A 3$ with other curves for the various stochastic models in Fig.7. It should be emphasized that the curve could be differentiated clearly from other curves because of its initial convex part.

Examples of the distribution fitting to

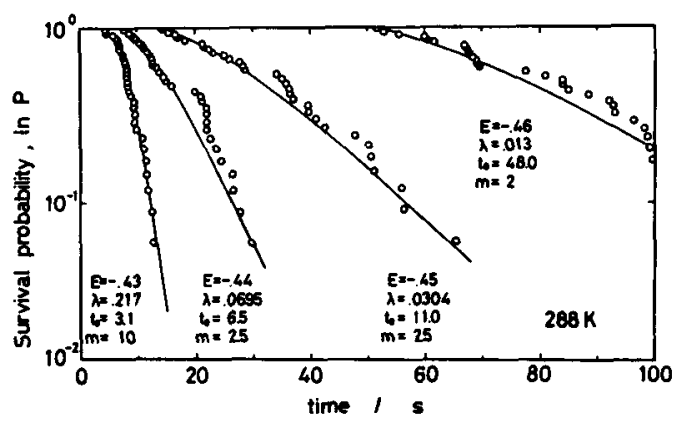

Fig.8 Distribution curves of induction time and their parameters -determined by the curve fitting.

the predicted curve for the parallel model were shown in Fig.8. The parameters indicated in the figure were decided by using an iteration method. It is clearly seen that the distribution of induction time is well fitted to the predicted convex curve, and the parameters decided are indicated in Fig.8. As summarized in Table 2, the parameter of $m$ decided at various potentials and temperatures is found to be in the range from 10 to 2, but almost 2. Thus, the analysis suggests that pit generation proceeds not by a single event, but by at least 2 coincidence events resulting in nucleation of embryo in the film and finally pit generation.

Effect of potential and temperature on the parameter

Two kinds of the relation had been reported for potentiaf dependence of pit generation rate, $\lambda, 2)$ and, in this experiment, the linear dependence is found to be expressed as

$$
\lambda=\alpha\left(\mathrm{E}^{-\mathrm{E}_{\text {crit }}}\right)
$$

where $\alpha$ and $E$ crit are a constant and the critical pitting potential, both of which are already defined in eq.(1) and decided in Fig.5. A similar equation had been reported by Broli et al. for induction time for pit generation of aluminum. It is noted that two different and independent experiments for determining pitting potential and for induction tine gives the consistent result for both

Table 2 Number of the parallel process incorporated in the pit generation.

Potenlial $(V) \quad-0.43 \quad-0.44 \quad-0.45 \quad-0.46-0.47 \quad-0.48-0.49-0.50$

Temp. (K)

\begin{tabular}{llllllll}
288 & 10 & 2.5 & 2.5 & 2 & & & \\
298 & & & 8 & 5 & 3.2 & 2 & \\
303 & & & & & 3 & 10 & 2 \\
\hline
\end{tabular}


parameters. Linear function of eq.(6) suggests that the film breakdown process is controlled not by the electrochemical, but an alternate mechanism such as the electro-restriction mechanism proposed by Sato ${ }^{2}$. On the other hand, incubation time obeys the exponential equation which is expressed as $\mathrm{t}_{0}=\mathrm{t}_{00} \exp (\gamma \mathrm{E})$

The exponential relation expressed by eq.(7) is explained by two alternate mechanisms. The first assumes the adsorption of chloride ions which are responsible for the formation of chlorocomplex causing the breakdown of the film. The rate of adsorption of anion on metal electrode is known to depend on potential in accord with the same exponential relation as eq.(7). It is reasonably assumed that incubation time corresponds to the time for accumulating chloride ions by a potential dependent adsorption to a critical concentration enough for the breakdown of the film. Second, it simply comes from the potential dependence of anodic dissolution during the pit growth, which is expressed as an exponential function of applied potential. The second mechanism is highly possible to operate in the pit generation process of aluminum, because the curves obtained in

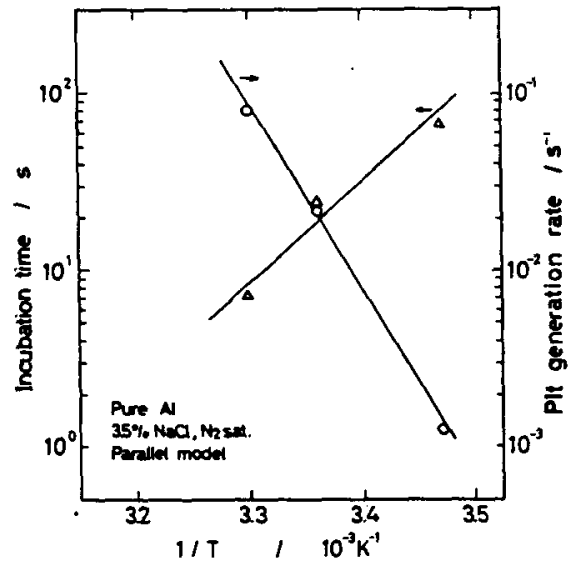

Fig.9 Temperature dependence of the pit generation rate and incubation time. the experiment could be properly simulated by a Monte Carlo method for the proposed mode1 10 . Effect of temperature on $\lambda$ and $t_{0}$ at the constant potential is summarized in Fig.9 and apparent activation energy for $\lambda$ and $t_{0}$ is found to be 304 and $109 \mathrm{~kJ}$ mol ${ }^{-1}$, respectively. These values suggests that both processes are controlled by a thermal activation process, and the film breakdown process proceeds not so easily compared with the anodic dissolution process.

\section{CONCLUSIONS}

Pit generation of pure aluminum in $3.5 \%$ $\mathrm{NaCl}$ solution was found to proceed as a plural combination of an elemental Poisson type stochastic process. The distribution of pitting potential and induction time was rationalized by the above stochastic model. Especially, the model was successful to simulate a very characteristic feature of the curve showing a convex in the initial part of the curve. It was concluded that at least 2 elemental stochastic processes incorporates to generate a pit.

\section{REFERENCES}

1) T. Shibata and T. Takeyama, Nature, 260, 315 (1976).

2) T. Shibata and T. Takeyama, Corrosion, 33, 243(1977).

3) T. H. Nguyen and R. T. Foley, J. Electrochem. Soc., 126, 1855 (1979).

4) T. Shibata and T. Takeyama, J. Japan Inst. Metals, 42, 743 (1978).

5) T. Shibata and T. Takeyama, J. Japan Inst. Metals, 43, 270 (1979).

6) T. Shibata, $17 \overline{2 n d}$ Meeting of The Electrochem. Soc., Extended Abstracts, Vo1.87-2, No.235 (1987).

7) T. Shibata and T. Takeyama, Proc. 8th Inter. Congr. Metallic Corrosion, p.146, DECHEMA, Frankfurt/Main (1981).

8) A. Broli, H. Holtan, and H. Sigurdsson, Werkst. Korros., 26, 629 (1975).

9) N. Sato, Electrochim. Acta, 16, 1683 (1971).

10) T. Shibata, Proc. Fushoku-Boshoku 88', B308, p.235, Japan Society of Corrosion Engineering, (1989).

11) T. Shibata, Proc. 6th Inter. Symp. on Passivity, to be published. 